\title{
Progressive Design Processes and Bounded Rational Designers
}

\author{
Jeffrey W. Herrmann \\ Associate Professor \\ Department of Mechanical Engineering and \\ Institute for Systems Research, \\ University of Maryland, \\ College Park, MD 20742 \\ e-mail: jwh2@umd.edu
}

This paper presents a method for assessing the quality of a progressive design process by measuring the profitability of the product that the process generates. The proposed approach uses separations, a type of problem decomposition, to model progressive design processes. The subproblems in the separations correspond roughly to phases in the progressive design processes. The proposed method simulates the choices of a bounded rational designer for each subproblem using different search algorithms. This paper presents a simple two-variable problem to help describe the approach and then applies the approach to assess motor design processes. Different types and versions of these search processes are considered to determine if the results are robust to the decisionmaking model. The results indicate that well-designed progressive design processes are the best way to generate profitable product designs. Methods for assessing the quality of engineering design processes can be used to guide improvements to engineering design processes and generate more valuable products. [DOI: 10.1115/1.4001902]

\section{Introduction}

This paper considers a certain class of engineering design processes that will be called progressive design. A progressive design process is an engineering design process that creates a product or system design through a series of distinct phases. (Thus, this term would not cover prototype-based design processes that iterate through generate-build-test cycles.) Each phase generates intermediate results by making decisions about different aspects of the design and generates increasingly detailed information. (The name reflects the similarity to a progressive die, which makes an increasingly complex part through a series of punches.) Pahl and Beitz [1], Asimow [2], Ullman [3], and Ulrich and Eppinger [4] are among those who presented progressive design processes; such processes are commonly found in industry $[5,6]$.

If product development organizations had a method for assessing the quality of engineering design processes, they could use this to guide improvements to their engineering design processes and generate more valuable products. In addition, one could assess the quality of design decision-making tools by considering how well they improve the entire engineering design process, not just one step. The purpose of the study described here is to develop such a method. The assessment method relies on new models of bounded rational designers. These models are searches, not random errors.

In addition to presenting the assessment method and the models of bounded rationality, this paper presents the results of applying this to a particular scenario and contributes some insights about design processes. First, this paper affirms the need for welldesigned progressive design processes. Drawing on the scenario, this paper highlights the impact of the objectives and constraints that are considered when making design decisions. Interestingly, considering profitability in every decision is not necessary to design a profitable product. Second, this paper indicates that, in the presence of bounded rationality, separating the profit maximization problem into a set of subproblems yields a better solution than trying to solve the profit maximization problem directly. Third, managers are not limited to consider cost and time metrics when assessing design processes.

Previous work on decision-making in engineering design has shown that design occurs via a series of decisions and that engi-

Contributed by the Design Theory and Methodology Committee of ASME for publication in the Journal of Mechanical Design. Manuscript received October 9 , 2009; final manuscript received May 12, 2010; published online July 21, 2010. Assoc. Editor: Jonathan Cagan. neering design organizations are decision-making systems $[7,8]$. Some decisions may be done sequentially while others occur concurrently, and different types of decision-making processes (such as designers who cooperate fully, designers who make isolated decisions, and sequential decision-making processes) can occur [9]. Research on this topic has focused primarily on the decisionmaking that sets parametric design variables within one phase of a system design project (e.g., the conceptual design of an aircraft or sizing a pressure vessel or electric motor).

An important development in recent years is the emergence of design for market systems approaches [10] and other work motivated by the decision-based design (DBD) framework [11], which includes enterprise models that add variables from the marketing and manufacturing domains to models with conceptual design variables and adapt existing decomposition techniques to solve them [12-14]. This perspective suggests that the profitability of a product (a measure of its performance in the marketplace) should be used to choose between different design options. This builds on the earlier suggestions of writers such as Smith and Reinertsen [15], who developed product profit models and used them to derive tradeoff rules to guide design decision-making. An important implication of this perspective is that it provides a single enterprise-level objective for evaluating the quality of product designs. A contribution of this paper is to provide a way to use profitability to assess the quality of an engineering design process.

This paper will continue by discussing the problem of designing design processes and proposing the use of separations to model design processes. Then, this paper will discuss bounded rationality and propose specific search algorithms as models for simulating the choices of a bounded rational designer. After these preliminaries, this paper will present the design process assessment method, present a simple example to help explain the method, and apply to the method to a motor design process scenario. After the presentation of the results of this scenario, the summary will share some insights and discuss the significance of the work.

\section{Designing Design Processes}

This study is concerned with the general problem of designing an engineering design process. An important aspect of this problem is the assessment of a design process. Methods for assessing design process time and cost have focused on the tasks that need to be performed. Methods for assessing design process quality have considered the decisions that are made. 
Various models of design processes consider the activities that need to be done, as in Gantt charts, the program evaluation and review technique (PERT) and critical path methods, integration definition for function modeling (IDEF), the design structure matrix, Petri nets, and signposting [16]. Such models have been used to estimate the cost and duration of design processes [17-22]. Eckert and Clarkson [23] described the practice of design planning, which focuses primarily on the tasks that need to be performed.

This study moves from studying the tasks that need to be performed to the decisions that need to be made. The design tasks are performed in order to make design decisions. Although task execution is important, it is also important to look at why those tasks are being performed. The structure of the design process is determined by the decisions that need to be made.

Some research has focused on assessing the quality of the design processes by evaluating the quality of the product designs that are generated. Chang and Ward [24] proposed a method for simultaneous decision-making of an engineering design team that focuses attention on the cost of waiting for information to make better decisions and the cost of making a wrong decision based on incomplete information. The simultaneous decision-making considered in their approach is not necessarily a good model of a progressive design process, however.

Krishnan et al. [25] considered the quality loss of engineering design processes, which they model as sequences of decisions made by different members of the engineering design team (see also Ref. [26]). The quality loss measures the distance of the resulting design from the optimal design (determined by making all of the decisions at the same time). They calculate the quality loss for different sequences of decisions in a dc motor design process. The quality loss of a sequence is determined by comparing each member's decision, which is constrained by the decisions make earlier in the sequence, to that member's optimal. Each member makes his decision once and has a different "optimal design" because they have different objectives. There is no bottom-line objective that all of the members seek to optimize.

Lewis and Mistree [9] considered the design of a pressure vessel and the design of a passenger aircraft. For both problems, they compared the quality of the solutions from different decisionmaking processes, which were modeled as two-player games. The objectives of the pressure vessel designers are to minimize weight and to maximize volume; the objectives of the passenger aircraft designers are to minimize deviations from aerodynamic and weight goals. In neither case is a more fundamental objective like profitability considered.

The approach described in this paper assumes that the objective of an engineering design process is to maximize the profitability of a new product. Different progressive design processes are modeled as separations, a special class of problem decompositions that are significantly different from typical decomposition-based design optimization methods [27].

Because humans are bounded rational decision-makers, this approach does not use optimization to solve the subproblems in the separation (as done in Ref. [27]). Instead, as Gurnani and Lewis [28] did, it models the designer's bounded rational design choices as a random process. In Ref. [28], the values chosen by the designer were determined by a random error term. In this work, however, the values are chosen by random search processes, as discussed later in this paper.

\section{Modeling Design Processes}

This study uses separations to model progressive design processes. A separation is a type of problem decomposition that divides a design optimization problem into a set of subproblems, solves each subproblem once, and produces a feasible solution without iterative cycles [27]. The ideal separation produces an optimal solution to the original problem. Unfortunately, not all separations do. Both separations and decomposition-based design optimization methods [29-36] replace a large design optimization problem with a set of subproblems. Separations are distinct because they do not solve a second-level problem to coordinate the subproblem solutions in an iterative manner.

In their work on sequential decision-making, Krishnan et al. [25] used the term decision order to describe a decision process in which each member of a cross-functional team makes his decision exactly once, in a predetermined. Such a sequence is one type of separation.

A leader-follower (or Stackelberg) game is also a simple separation. In this scenario, one player makes his decision and then the other player makes his decision. Lewis and Mistree [9] studied this as one model of collaborative decision-making.

Previous work evaluated the quality of different separations by using optimization to solve each subproblem [27]. If the subproblems are correctly formulated, the separation yields an optimal solution. The quality of approximate separations depends on the constraints and objectives used in the subproblems.

This paper pursues the idea suggested in Ref. [27] that separation could be used to model an engineering design process. In particular, it studies separations corresponding to different types of progressive design processes. If decision-makers always optimized, then separation should, except under certain conditions, lead to a quality loss $[25,27]$. Because real-world decision-makers cannot optimize, the proposed method will evaluate these separations by using a model of a bounded rational designer to find solutions to each subproblem.

\section{Bounded Rationality}

Most studies of engineering decision-making processes assume that each engineer optimizes his objective function. For example, to design a pressure vessel, Lewis and Mistree [9] formulated the problem as a two-player game in which the first player, who wishes to maximize the volume, controls the diameter and length and the second player, who wishes to minimize the weight, controls the thickness. Chen and $\mathrm{Li}$ [37] modeled the interactions of teams in concurrent parametric design under the assumption that each team optimized their aspect of the complete design. In the decision orders of Krishnan et al. [25], each team member selects values for their design variables in order to optimize their individual objective function.

It is well-known that real-world decision-makers cannot optimize because of limits on their problem-solving capacity. This concept is known as bounded rationality [38]. Bounded rationality reflects the observation that in most real-world cases, decisionmakers have limited information and limited computational capabilities for finding and evaluating alternatives and choosing among them [39-41]. A decision-maker cannot perfectly evaluate the consequences of the available choices. This prevents complete and perfect optimization. The study of procedures besides optimization to choose between alternatives has been defended by appealing to bounded rationality, as in the case of pairwise comparisons of a finite set of alternatives [42].

Satisficing and fast and frugal heuristics are two models of bounded rationality [40]. Bounded rational decision-makers may search until they find something that meets their requirements (satisficing) or they may use fast and frugal heuristics that search a limited set of objects and information and make choices using rules that are easy to compute (and, therefore, quick). This study consider only this second type of bounded rationality and leaves satisficing to future work.

Gurnani and Lewis [28] studied collaborative, decentralized design processes in which the models of the individual decisionmakers (the designers) were based on the ideas of bounded rationality. In their model, the value chosen by each designer was determined by randomly sampling from a distribution around the (locally) "optimal" solution. This model is meant to represent the mistakes that designers make due to bounded rationality. Their results show that incorporating bounded rationality leads to more 
desirable solutions in a collaborative, decentralized design process in which the designers had different objectives and no way to coordinate their activities.

This paper will take a different approach to modeling bounded rationality. To motivate this approach, this section will first consider models of organizational decision-making and problemsolving and then consider cognitive models of human problemsolving.

In each phase of a progressive design process, some aspect of the design needs to be determined. That is, the designer must make a decision. In practice, the designer does not have the complete information about the available alternatives and their performance on the relevant attributes. Thus, the decision-maker must employ some process for generating and evaluating alternatives. (Note that this study is considering situations that involve searching for alternatives and the information needed to evaluate them, not simple choices or perfect information games and puzzles such as chess and Sudoku.)

The incremental decision process model describes the activities that occur during decision-making processes in product development, facility design, procurement, and policy setting [43]. This decision-making process (also known as the strategic decisionmaking process) is not a simple sequence of tasks but involves iterating between the following types of activities: recognition, diagnosis, search, screen, design, judgment, analysis, bargaining, and authorization.

March and Simon [41] described the general characteristics of human problem-solving in organizational decision-making. The first characteristic is that making a complex decision involves making a large number of small decisions. The second characteristic is that problem-solving has a hierarchical structure in which solving any problem goes through phases that, in turn, require solving more detailed subproblems. The general concept of separation is related to these two characteristics. The third characteristic is that problem-solving consists of searching for possible solutions (compare Ref. [44]). The fourth characteristic is that problem-solving includes screening processes that evaluate the solutions that are found. The fifth characteristic is that problemsolving has not only random components (such as finding and evaluating solutions) but also a procedural structure that allows it to yield good solutions. The proposed model of a bounded rational designer is motivated by these last three characteristics.

Wang and Chiew [45] described human problem-solving as a higher-layer cognitive process that can be considered as a search process although it requires other cognitive processes such as abstraction, analysis, synthesis, and decision-making. Their model of a generic problem-solving process is a search that iteratively generates and evaluates potential solutions.

Thus, decision-making in the context of product development is equivalent to problem-solving by organizations and individual humans, and, furthermore, such problem-solving is essentially a search.

\section{Modeling Bounded Rationality}

An important aspect of bounded rationality is that the resources and time available for problem-solving are limited. Consequently, the proposed model of a bounded rational designer incorporates limits that will constrain the amount of time available for the search and the accuracy of the evaluation of a solution.

Therefore, the design process assessment method will use a class of search algorithms that identify and evaluate solutions to model the choices of a bounded rational designer. Each search has random components (either randomly selecting a solution or randomly moving to a point near the existing solution) and the evaluation of a solution has a random error that represents inaccuracies due to limited time and knowledge. The procedural structure of the model attempts to compensate for this randomness by keeping track of the "best" solution found so far. (The best requires quotes because the evaluation of the solution may be inaccurate.) Finally, the search is limited to a fixed number of solutions.

This study considers two types of searches: random sampling and local search. Within each type, the search is characterized by two parameters: $N$ is the search effort and $E$ is the maximum relative error. Let $X$ be the vector of design variables with lower and upper bounds $L$ and $U$. In addition, the search may need to satisfy one or more constraints $C$ in addition to the lower and upper bounds. Let $f(X)$ be the objective function.

The random sampling search works as follows.

Do the following step $N$ times: Randomly select $X$ between $L$ and $U$. If $X$ is not feasible with respect to $C$ then "repair" $X$ by projecting it into the feasible space and replacing $X$ with the repaired solution. Select the relative error $\varepsilon$ from a uniformly distributed random variable with the range $[-E, E]$. Set $g(X)=(1$ $+\varepsilon) f(X)$. If $g(X)$ is the best function evaluation found so far, keep $X$ as the best solution found so far.

The local search works as follows.

Randomly select $X$ between $L$ and $U$. If $X$ is not feasible with respect to $C$ then repair $X$ by projecting it into the feasible space and replacing $X$ with the repaired solution. Select the relative error $\varepsilon$ from a uniformly distributed random variable with the range $[-E, E]$. Set $g(X)=(1+\varepsilon) f(X)$. Do the following step $N$ times: Let $D=(1 / 20)(U-L)$. Randomly select a step $S$ between $-D$ and $D$ and let the new solution $Y=X+S$. If $Y$ is not feasible with respect to $C$ then repair $Y$ by projecting it into the feasible space and replacing $Y$ with the repaired solution. Select the relative error $\varepsilon$ from a uniformly distributed random variable with the range $[-E, E]$. Set $g(Y)=(1+\varepsilon) f(Y)$. If $g(Y)$ is better than $g(X)$, then set $X=Y$.

Both searches return $X$, the best solution found so far. After the search ends $f(X)$, the true value of the objective function at this point will be calculated for evaluation purposes.

It is important to note that these searches are meant to represent a bounded rational designer. These algorithms should not be compared with state-of-the-art techniques for solving design optimization problems. Like the work in Ref. [28], the proposed design assessment method is modeling the designer's bounded rational design choices as a random process. In Ref. [28], the values chosen by the designer were determined by a random error term. In the proposed approach, on the other hand, the values are chosen by a random search process. To model a bounded rational designer who is using a type of fast and frugal heuristic, these searches have simple rules to stop the search (when the number of solutions evaluated equals $N$ ) and to choose a solution (whether it is better than the best found so far).

\section{Design Process Assessment Method}

This section describes the design process assessment method. This method is meant to be used by a product development organization that has identified two or more design processes and desires to compare them.

1. For each design process under consideration, determine the key decisions that are made and the flow of information between them, which also specifies the precedence constraints between these decisions.

2. For each decision in each design process, determine the variables that are set by the decision, the constraints that limit the values of the variables and the objective used to make the decision.

3. For each decision in each design process, build an appropriate simulation model of the bounded rational designer making that decision by taking into account the information available from previous decisions in that process and the variables, constraints, and objective of that decision.

4. For each design process under consideration, run the simu- 


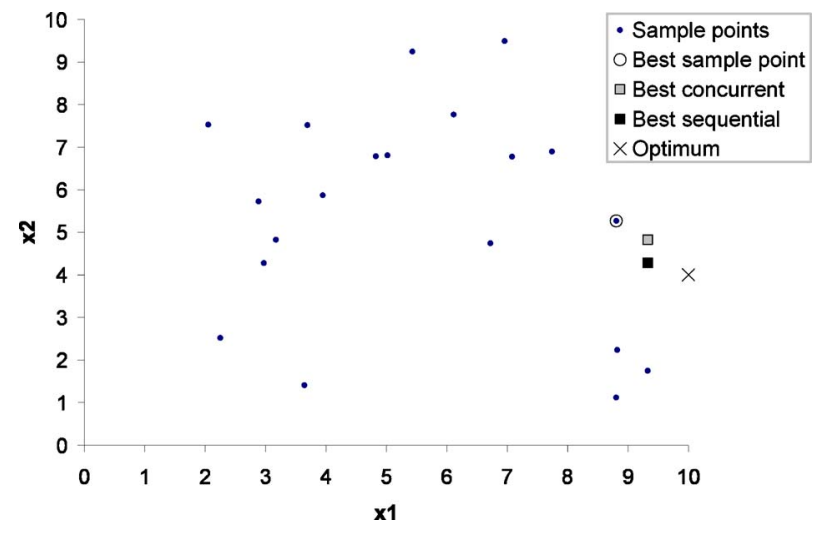

Fig. 1 Sample points and solutions for two-variable example

lation models for the decisions in that process in order to estimate the expected product quality for that process.

\section{Two-Variable Example}

For instance, consider three design processes for a simple twovariable design problem. The two design variables $x_{1}$ and $x_{2}$ have the bounds $1 \leq x_{1} \leq 10$ and $1 \leq x_{2} \leq 10$ The objective function to minimize is $f\left(x_{1}, x_{2}\right)=x_{2} / x_{1}+\frac{1}{20}\left(x_{2}-5\right)^{2}$. The optimal value occurs at $\left(x_{1}, x_{2}\right)=(10,4)$ with $f(10,4)=0.45$. This point is marked by with a black $\mathrm{X}$ in Fig. 1 .

The first process considers both variables simultaneously. A search generated 20 points by sampling from uniform distributions for both $x_{1}$ and $x_{2}$ (both on the interval $[1,10]$ ). This yielded the points in Table 1 and they are also marked by small dark circles in Fig. 1. The values of $f\left(x_{1}, x_{2}\right)$ are given as well and the best point is $(8.8046,5.2671)$ with $f(8.8046,5.2671)=0.6018$. This point is circled in Fig. 1.

The second process employs two distinct but concurrent searches: one for $x_{1}$ and one for $x_{2}$. The search for $x_{1}$ seeks to minimize $1 / x_{1}$ and the search for $x_{2}$ seeks to minimize $\left(x_{2}-5\right)^{2}$. For each variable, the search used the same 20 samples that were generated before. The best $x_{1}=9.3261$, and the best $x_{2}=5.2671$. This point is marked with a gray square in Fig. 1 and $f(9.3261,5.2671)=0.5192$.

Table 1 Sample points, for example

\begin{tabular}{cccc}
\hline \hline Point & $x_{1}$ & $x_{2}$ & $f\left(x_{1}, x_{2}\right)$ \\
\hline 1 & 6.9567 & 9.4910 & 2.3728 \\
2 & 6.7216 & 4.7429 & 0.7089 \\
3 & 7.7413 & 6.8977 & 1.0711 \\
4 & 2.0509 & 7.5269 & 3.9894 \\
5 & 7.0820 & 6.7763 & 1.1146 \\
6 & 2.8837 & 5.7263 & 2.0121 \\
7 & 3.9476 & 5.8739 & 1.5262 \\
8 & 2.2510 & 2.5185 & 1.4267 \\
9 & 3.6916 & 7.5195 & 2.3543 \\
10 & 8.8191 & 2.2350 & 0.6357 \\
11 & 5.4328 & 9.2444 & 2.6023 \\
12 & 4.8252 & 6.7868 & 1.5662 \\
13 & 2.9704 & 4.2774 & 1.4661 \\
14 & 6.1129 & 7.7658 & 1.6529 \\
15 & 5.0185 & 6.8124 & 1.5217 \\
16 & 3.1707 & 4.8280 & 1.5242 \\
17 & 8.8046 & 5.2671 & 0.6018 \\
18 & 9.3261 & 1.7465 & 0.7165 \\
19 & 8.8042 & 1.1194 & 0.8801 \\
20 & 3.6420 & 1.4091 & 1.0316 \\
\hline \hline
\end{tabular}

Table 2 Bounds on product design variables

\begin{tabular}{lccc}
\hline \hline Variable & Definition & Bounds & Units \\
\hline$N_{c}$ & Turns of wire (armature) & {$[100,1500]$} & turns \\
$N_{s}$ & Turns of wire (stator), per pole & {$[1,500]$} & turns \\
$A_{a w}$ & Cross sectional area of armature wire & {$[0.01,1.0]$} & $\mathrm{mm}^{2}$ \\
$A_{s w}$ & Cross sectional area of stator wire & {$[0.01,1.0]$} & $\mathrm{mm}^{2}$ \\
$r_{o}$ & Outer radius (stator) & {$[0.01,0.1]$} & $\mathrm{m}$ \\
$t_{s}$ & Thickness (stator) & {$[0.0005,0.01]$} & $\mathrm{m}$ \\
$I$ & Electric current & {$[0.1,6]$} & $\mathrm{A}$ \\
$L$ & Stack length & {$[0.01,0.2]$} & $\mathrm{m}$ \\
\hline \hline
\end{tabular}

The third process is a sequence of two searches: the first for $x_{1}$ and the second for $x_{2}$. The search for $x_{1}$ seeks to minimize $1 / x_{1}$, and the search for $x_{2}$ seeks to minimize $f\left(x_{1}, x_{2}\right)$. For each variable, the searches used the same 20 samples that were generated before. The best $x_{1}=9.3261$. Given this value for $x_{1}$, the best $x_{2}$ $=4.2774$. This point is marked with a black square in Fig. 1, and $f(9.3261,4.2774)=0.4848$.

Note that, for a bounded rational decision-maker, the decisionmaking processes (separations) that divided the original optimization problem into two subproblems found better solutions than the process that considered both variables at the same time. The solution of the concurrent decision-making process was not as good because the search for $x_{2}$ considered only half of the objective function (because there was no value of $x_{1}$ present). The solution of the sequential decision-making process was closer to the optimal solution.

\section{Motor Design Process Example}

The process of designing a universal electric motor will be used to demonstrate the proposed design process assessment method. The motor design example was originally developed by Simpson $[46,47]$ to demonstrate new techniques in product family design. The following example ignores the product family aspect, considers only a single motor design that should meet given power and torque requirements, and uses a different cost function. In this example, a product development organization is considering three alternatives to its current design process. The first two alternatives would change the criteria used to select a motor design; the third alternative would combine motor design and pricing.

Before discussing the design processes, this section will briefly describe the motor performance and profitability models that will be used.

The universal electric motor design problem includes eight design variables, four customer attributes, 23 intermediate engineering attribute calculations, six constraints, and seven fixed engineering parameters. The derivations of the equations and other background information on universal electric motors can be found in Refs. $[46,47]$. The nomenclature and equations for the design variables, fixed model parameters, customer attributes, and constraints are listed below. Appendix A lists the intermediate engineering attributes. Table 2 lists the product design variables and their bounds. The price $p$ also must be determined.

The four customer attributes are the torque $T$ (in $\mathrm{Nm}$ ), the power $P$ (in watts), the efficiency $\eta$, and the mass $M$ (in $\mathrm{kg}$ ). They are calculated from the design variables and the engineering attributes as follows:

$$
\begin{gathered}
T=K \varphi I \\
P=P_{\text {in }}-P_{\text {out }} \\
\eta=P / P_{\text {in }} \\
M=M_{w}+M_{s}+M_{a}
\end{gathered}
$$


Table 3 Design processes and separations

\begin{tabular}{|c|c|}
\hline Design process & Subproblem(s) in the separation \\
\hline A & $\begin{array}{l}\text { 1. Set design variables to minimize cost. } \\
\text { 2. Set price to maximize profit. }\end{array}$ \\
\hline B & $\begin{array}{l}\text { 1. Set design variables to maximize efficiency. } \\
\text { 2. Set price to maximize profit. }\end{array}$ \\
\hline $\mathrm{C}$ & $\begin{array}{l}\text { 1. Set design variables to maximize utility. } \\
\text { 2. Set price to maximize profit. }\end{array}$ \\
\hline $\mathrm{D}$ & 1. Set design variables and price to maximize profit. \\
\hline
\end{tabular}

The product's power should equal $300 \mathrm{~W}$ and its torque should equal $0.05 \mathrm{Nm}$.

The demand model is a logit model with independent and identically distributed error terms. (For more about this type of model in particular and discrete choice analysis in general, see Ref. [48].) In this aggregate model, demand depends on the additive utility function $\nu$, which is calculated from the utility functions for the mass, efficiency, and price attributes. The total demand $(d)$ is the population size $(s)$ multiplied by the probability that a consumer will select this design. In this scenario, $s=1 \times 10^{6}$.

$$
\begin{gathered}
\Psi_{1}(M)=0.5(1-M) \\
\Psi_{2}(\eta)=\eta-0.5 \\
\Psi_{3}(p)=\frac{25-4 p}{15} \\
v=\Psi_{1}(M)+\Psi_{2}(\eta)+\Psi_{3}(p) \\
d=s e^{v} /\left(1+e^{v}\right)
\end{gathered}
$$

The cost model is a modification of the cost equations that were originally derived in Wassenaar and Chen [49]. The unit cost $C$ depends on the mass of the major components, the material costs, and an overhead rate.

$$
C=\frac{10}{7}\left(M_{w} C_{\text {copper }}+\left(M_{s}+M_{r}\right) C_{\text {steel }}\right)
$$

The profit $\Pi$ of a motor design is a function of the demand $(d)$, the price $(p)$, and the unit cost.

$$
\Pi=d(p-C)
$$

The constraints on the power and torque reduce the search space as follows. Given values for the first six design variables (the independent design variables) it is possible to find values for $I$ and $L$ so that product satisfies the power and torque requirements. (Appendix B derives the required equations.)

The performance-and-cost utility function $u$ is related to the demand model

$$
u=\eta-\frac{1}{2} M-\frac{4}{15} C
$$

Step 1. Determine the key decisions and information flow for each design process under consideration. In this example, a product development organization currently designs the motor first and then determines the price. (For clarity, the current design process will be called process A.) The firm is considering two alternatives (called process $\mathrm{B}$ and process $\mathrm{C}$ ) that use this same structure and a third alternative (called process D) that would combine these two phases into one phase that determines the motor design and the price simultaneously. Table 3 summarizes the design processes.

Step 2. Determine the variables, constraints, and objective used in each decision. In processes A-C, the variables of the first decision are the six independent design variables. The key constraints are the power and torque constraints. (The relationships between the design variables and the attributes are also relevant.) In process $A$, the objective of the first decision is to minimize the
Table 4 Results of local search for different values of search effort. Maximum error $=0 ; 90 \%$ confidence intervals on the average profit.

\begin{tabular}{cll}
\hline \hline Process & \multicolumn{1}{c}{500} & \multicolumn{1}{c}{1000} \\
\hline $\mathrm{A}$ & $3,613,000 \pm 448,000$ & $3,524,000 \pm 471,000$ \\
$\mathrm{~B}$ & $3,520,000 \pm 129,000$ & $3,528,000 \pm 97,000$ \\
$\mathrm{C}$ & $4,518,000 \pm 15,000$ & $4,522,000 \pm 7,000$ \\
$\mathrm{D}$ & $4,301,000 \pm 308,000$ & $4,428,000 \pm 128,000$ \\
\hline \hline
\end{tabular}

cost of the motor. In process $\mathrm{B}$, the objective of the first decision is to maximize the motor efficiency. In process $C$, the objective of the first decision is to optimize $u$ the utility function that includes both performance and unit cost.

In processes $\mathrm{A}-\mathrm{C}$, the only variable in the second decision is the price. The price must be positive, and the objective is to maximize the profitability of the motor. (The relationships between the price, attributes, demand, and profitability are also relevant.)

Process D has only one decision. The variables of this decision are the six independent design variables and the price. The relevant constraints are the power and torque constraints and the price must be positive. The objective is to maximize the profitability of the motor. (The relationships between the design variables, the attributes, the price, the demand, and the profitability are also relevant.)

Step 3. Build an appropriate simulation model for each decision. To assess these design processes and determine the robustness of the evaluation, this study modeled the bounded rational designer using both types of search (sampling and local search), two levels of search effort (500 and 1000 solutions per search) and three values for maximum error $(0 \%, 1 \%$, and $10 \%)$. The maximum error and search effort are the same across all of the subproblems in a given separation. The levels of search effort were chosen after preliminary results showed that the profitability of the solutions continued to increase significantly until about 500 samples; after that the profitability increased more slowly.

Step 4. Run the simulation models. For each combination of separation, search type, search effort, and maximum error, 25 replications of the search were run. The results are described in Sec. 5 . The best solution found in any search had a profit of $\$ 4,524,000$.

\section{Results}

The results show that some design processes yield much better solutions than others, as shown in Tables 4-9. (The profit values are rounded to the nearest $\$ 1000$.) Process $\mathrm{C}$ (maximizing utility) yields more profitable solutions than any other process. This result is statistically significant across the different models of bounded rationality (local search and sampling), different levels of search effort, and different levels of maximum error.

Process B (maximizing efficiency) is clearly the worst process, and process $\mathrm{D}$ (maximizing profit all-at-once) is somewhat better than process A (minimizing unit cost) but the differences between the processes vary across the different searches.

Table 5 Results of local search for different values of search effort. Maximum error $=1 \% ; 90 \%$ confidence intervals on the average profit.

\begin{tabular}{cll}
\hline \hline Process & \multicolumn{1}{c}{500} & \multicolumn{1}{c}{1000} \\
\hline A & $3,644,000 \pm 420,000$ & $3,543,000 \pm 514,000$ \\
B & $3,188,000 \pm 1,845,000$ & $3,430,000 \pm 683,000$ \\
C & $4,482,000 \pm 54,000$ & $4,506,000 \pm 28,000$ \\
D & $4,230,000 \pm 306,000$ & $4,356,000 \pm 164,000$ \\
\hline
\end{tabular}


Table 6 Results of local search for different values of search effort. Maximum error $=10 \%$; $90 \%$ confidence intervals on the average profit.

\begin{tabular}{cll}
\hline \hline Process & \multicolumn{1}{c}{500} & \multicolumn{1}{c}{1000} \\
\hline A & $3,545,000 \pm 531,000$ & $3,520,000 \pm 499,000$ \\
B & $2,880,000 \pm 2,399,000$ & $3,153,000 \pm 1,899,000$ \\
C & $4,254,000 \pm 280,000$ & $4,343,000 \pm 225,000$ \\
D & $3,977,000 \pm 800,000$ & $4,167,000 \pm 290,000$ \\
\hline \hline
\end{tabular}

Thus, in this example, the product development organization should adopt maximizing utility as its objective when setting the design variables in the first phase of its progressive design process. They should not try to set the design variables and the price simultaneously.

\section{Summary and Conclusions}

This paper presented a method for assessing the quality of progressive design processes. This method can be used to select between alternative design processes and to guide improvements to a design process. The proposed approach uses separations, a type of problem decomposition, to model progressive design processes. The subproblems in the separations correspond roughly to phases in the progressive design processes. The choices of a bounded rational designer for each subproblem are simulated using different search algorithms.

This study considered two types of searches: a sampling search that randomly selects feasible solutions and keeps the best found so far and a local search that randomly selects a solution near the current solution and moves to the new solution if it is better than the current solution.

The results show that, for the motor design process example, a design process that maximizes a performance-and-cost utility

Table 7 Results of sampling for different values of search effort. Maximum error $=0 ; 90 \%$ confidence intervals on the average profit.

\begin{tabular}{cll}
\hline \hline Process & \multicolumn{1}{c}{500} & \multicolumn{1}{c}{1000} \\
\hline A & $3,933,000 \pm 609,000$ & $4,014,000 \pm 514,000$ \\
B & $3,538,000 \pm 287,000$ & $3,400,000 \pm 680,000$ \\
C & $4,408,000 \pm 93,000$ & $4,441,000 \pm 70,000$ \\
D & $3,992,000 \pm 366,000$ & $4,110,000 \pm 305,000$ \\
\hline
\end{tabular}

Table 8 Results of sampling for different values of search effort. Maximum error $=1 \% ; 90 \%$ confidence intervals on the average profit.

\begin{tabular}{cll}
\hline \hline Process & \multicolumn{1}{c}{500} & \multicolumn{1}{c}{1000} \\
\hline A & $3,927,000 \pm 613,000$ & $4,009,000 \pm 513,000$ \\
B & $3,373,000 \pm 1,128,000$ & $3,505,000 \pm 432,000$ \\
C & $4,397,000 \pm 102,000$ & $4,435,000 \pm 75,000$ \\
D & $3,989,000 \pm 368,000$ & $4,109,000 \pm 304,000$ \\
\hline \hline
\end{tabular}

Table 9 Results of sampling for different values of search effort. Maximum error $=10 \%$; $90 \%$ confidence intervals on the average profit.

\begin{tabular}{cll}
\hline \hline Process & \multicolumn{1}{c}{500} & \multicolumn{1}{c}{1000} \\
\hline A & $3,970,000 \pm 539,000$ & $4,001,000 \pm 519,000$ \\
B & $3,157,000 \pm 1,304,000$ & $3,206,000 \pm 1,284,000$ \\
C & $4,289,000 \pm 188,000$ & $4,359,000 \pm 165,000$ \\
D & $3,857,000 \pm 403,000$ & $4,011,000 \pm 356,000$ \\
\hline \hline
\end{tabular}

function first and then sets the price generates more profitable solutions than a design process that attempts to solve the profit maximization problem all-at-once. Design processes that minimize cost or maximize efficiency perform poorly. The relative performance is robust and holds across a range of different variations in the model of the bounded rational designer. In addition, the results show that the quality of different types of searches depends on the presence of error in the evaluation of a solution.

Note that these results are not meant to imply that designers should use any of these algorithms explicitly to solve design optimization problems. They are models of how a bounded rational designer makes choices and their purpose is to help assess the quality of different design processes.

This paper began by considering how an organization could assess the quality of engineering design processes and discussed how the emergence of the design for market systems approach suggests that profitability is an appropriate objective function for evaluating design process quality. The approach presented here uses this objective unlike previous approaches that focused on less-fundamental objectives. Moreover, instead of assuming that designers optimize, this approach starts with a more realistic position that designers are bounded rational decision-makers. It uses a random process to model their design choices as part of the assessment procedure. The results show that some (but not all) progressive design processes lead to designs that are more profitable than those generated by attempts to maximize profitability by considering all of the design variables simultaneously.

These results, therefore, provide some insights into the problem of assessing design processes in general and the benefits of progressive design processes in particular.

First, the structure and objectives of the decisions in a design process affect the quality of a design process (measured by the expected profitability of the product that the process generates). Thus, organizations should assess process quality to guide the overall structure before considering the time and cost of the tasks to be performed within the structure.

Second, although there is no automated way to separate product design into subproblems, one strategy is to examine the coupling between the decision variables. In many cases, the variables are loosely coupled [50]. There will be tightly coupled groups of variables that should be set at the same time but the different groups can be separated into different decisions. For instance, in the motor design process example, the design variables are tightly coupled to each other but loosely coupled with the price. Thus, it would be difficult to design a design process that sets only some of the design variables but not the others.

Third, the objectives and constraints of the decision-making activities in a progressive design process are influential factors. Therefore, modifying these can significantly improve or degrade the quality of a progressive design process. Because engineers can have different views of what they are trying to do [51], organizations should make sure that everyone understands the objective function that they are trying to optimize and the constraints that must be satisfied.

Finally, because human decision-makers have limitations and cannot optimize, well-designed progressive design processes are the best way to generate profitable product designs. Trying to set everything all-at-once (by formulating enterprise models) will lead to problems that are too large to solve well. The large solution space of the single-stage optimization problem makes it difficult for the bounded rational decision-maker to approach an optimal solution.

This research, unlike previous works, is ultimately concerned with the overall engineering design process, not with just the decision-making within a phase of the process. Although progressive design processes for complex products are extremely complicated, it is hoped that models that are similar to the ones presented here could be used to help organizations improve their design 
processes. Models that include the key decision-making activities and most important design variables may provide useful insights.

The separations studied in Ref. [27] were used to solve design optimization problems, and optimization was used to solve each subproblem. Separations reduced the computational effort and some separations generated solutions that were nearly as profitable as those found by solving the all-at-once formulation.

In this paper, separations are used as models of progressive design processes and random searches (rather than optimization) are used to find solutions to the subproblems. The results in this paper show that a bounded rational designer will generate more profitable designs by using a sequential decision-making process.

This paper builds on two important branches of the study of design decision-making. The first branch has developed the methods for profit maximization in design and the second branch has studied the process of how decisions are made. The approach presented here is an attempt to connect these two different branches.

This paper contributes to our knowledge about design decisionmaking by proposing specific random search algorithms as models for the choices of a bounded rational designer (unlike Gurnani and Lewis [28], who used a random error term). This idea reflects the importance of search in design (compare Ref. [50]). More work is needed to consider other models based on satisficing or other types of fast and frugal heuristics.

Previous work on assessing the quality of design processes did not have an overarching objective for discussing the quality of different decision-making processes. The emerging area of design for market systems (building on the DBD framework) provides such an objective (profitability) and motivates recovering and reassessing these ideas within this new context.

A second contribution of this paper is to show that, when a bounded rational designer seeks profitable designs, separating the profit maximization problem into a set of subproblems yields a better solution than attempting to solve the profit maximization problem directly. Thus, using an appropriate progressive design process is a reasonable design for market systems strategy.

Thus, this paper contributes a new perspective on the design for market systems approach by endorsing the consideration of profitability as a fundamental objective but proposing that this objective be used to evaluate progressive design processes (instead of formulating enterprise models).

\section{Acknowledgment}

Peyman Karimian wrote some of the computer code used in this work. The author also appreciates the useful comments of anonymous reviewers on previous versions of this paper.

\section{Appendix A: Engineering Parameters and Attributes}

\section{A.1 Engineering Parameters}

Length of air gap $l_{g}=7.0 \times 10^{-4} \mathrm{~m}$

Terminal voltage $V_{t}=115 \mathrm{~V}$

Resistivity of copper $\rho=1.69 \times 10^{-8} \Omega \mathrm{m}$

Permeability of free space $\mu_{o}=4 \pi \times 10^{-7} \mathrm{H} / \mathrm{m}$

Number of stator poles $p_{s t}=2$

Cost of copper $C_{c}=2.2051 \$ / \mathrm{kg}$

Cost of steel $C_{s}=0.882 \$ / \mathrm{kg}$

Density of copper $\delta_{c}=8960 \mathrm{~kg} / \mathrm{m}^{3}$

Density of steel $\delta_{s}=7861.09 \mathrm{~kg} / \mathrm{m}^{3}$

Relative permeability of steel (dimensionless) $\mu_{\text {steel }}=1000$

\section{A.2 Engineering Attributes}

Magnetizing intensity (A turns/m)

$$
H=N_{c} I /\left(l_{c}+l_{r}+2 l_{g}\right)
$$

Mean path length within the stator $(\mathrm{m})$

$$
l_{c}=\pi\left(2 r_{o}+t_{s}\right) / 2
$$

Diameter of armature $(\mathrm{m}) l_{r}=2\left(r_{o}-t_{s}-l_{g}\right)$

Input power $(\mathrm{W}) P_{\text {in }}=V_{t} I$

Power losses due to copper and brushes (W)

$$
P_{\text {out }}=I^{2}\left(R_{a}+R_{s}\right)+2 I
$$

Armature wire length $(\mathrm{m}) l_{a w}=\left(2 L+2 l_{r}\right) N_{c}$

Stator wire length $(\mathrm{m}) l_{s w}=p_{s t}\left(2 L+4\left(r_{o}-t_{s}\right)\right) N_{s}$

Armature wire resistance $(\Omega) R_{a}=\rho l_{a w} / A_{a w} \times 10^{6}$

Stator wire resistance $\Omega R_{s}=\rho l_{s w} / A_{s w} \times 10^{6}$

Mass windings $(\mathrm{kg}) M_{w}=\left(l_{a w} A_{a w}+l_{s w} A_{s w}\right) \delta_{c} \times 10^{-6}$

Mass of stator $(\mathrm{kg}) M_{s}=\pi L\left(r_{o}^{2}-\left(r_{o}-t_{s}\right)^{2}\right) \delta_{s}$

Mass of armature $(\mathrm{kg}) M_{a}=\pi L\left(r_{o}-t_{s}-l_{g}\right)^{2} \delta_{s}$

Motor constant (dimensionless) $K=N_{c} / \pi$

Magneto magnetic force (A turns) $\mathfrak{I}=N_{s} I$

Magnetic flux $(\mathrm{Wb}) \varphi=\Im / \Re$

Total reluctance (A turns/Wb) $\mathfrak{R}=\mathfrak{R}_{s}+\mathfrak{R}_{a}+2 \mathfrak{R}_{g}$

Stator reluctance (A turns/Wb) $\Re_{s}=l_{c} /\left(2 \mu_{\text {steel }} \mu_{o} A_{s}\right)$

Armature reluctance (A turns/Wb)

$$
\Re_{a}=l_{r} /\left(\mu_{\text {steel }} \mu_{o} A_{a}\right)
$$

Reluctance of one air gap (A turns/Wb)

$$
\mathfrak{R}_{g}=l_{g} /\left(\mu_{o} A_{g}\right)
$$

Cross sectional area of stator $\left(\mathrm{m}^{2}\right) A_{s}=t_{s} L$

Cross sectional area of armature $\left(\mathrm{m}^{2}\right) A_{a}=l_{r} L$

Cross sectional area of air gap $\left(\mathrm{m}^{2}\right) A_{g}=l_{r} L$

\section{Appendix B: Derivation of Dependent Design Variables}

First, define the quantities $Z$ and $W$, which depend upon the independent design variables, the engineering parameters, and the given torque.

$$
\begin{gathered}
Z=\frac{\pi\left(2 r_{0}+t_{s}\right)}{4 \mu_{\text {steel }} \mu_{0} t_{s}}+\frac{1}{\mu_{\text {steel }} \mu_{0}}+\frac{7 \times 10^{-4}}{\mu_{0}\left(r_{0}-t-7 \times 10^{-4}\right)} \\
W=\frac{T \pi Z}{N_{C} N_{S}}
\end{gathered}
$$

Because the total reluctance $\Re=Z / L$, the formula for torque can be rearranged to give the following relationship between $L$ and $I$ :

$$
L=\frac{W}{I^{2}}
$$

The relationships for $P_{\text {in }}$ and $P_{\text {out }}$ can be used to derive the following expression:

$$
P=115 I-\left(I^{2}\left(R_{a}+R_{S}\right)+2 I\right)
$$

Including the definitions of $R_{a}$ and $R_{S}$ from Appendix $\mathrm{A}$ and defining the quantities $C_{1}, C_{2}, C_{3}$, and $C_{4}$ (which also depend only upon the six independent design variables) provides an expression for power in terms of $I$ and $L$.

$$
\begin{gathered}
C_{1}=1.69 \times 10^{-8} \times 10^{6}=1.69 \times 10^{-2} \\
C_{2}=\frac{2 N_{c}}{A_{a w}}+\frac{4 N_{s}}{A_{s w}} \\
C_{3}=4\left(r_{0}-t-7 \times 10^{-4}\right) \frac{N_{C}}{A_{a w}} \\
C_{4}=8\left(r_{0}-t\right) \frac{N_{S}}{A_{s w}} \\
P=113 I-C_{1} I^{2}\left(C_{2} L+C_{3}+C_{4}\right)
\end{gathered}
$$

Because $P$ must equal 300, substituting Eq. (B2) into Eq. (B5) yields a quadratic function of $I$. 


$$
C_{1}\left(C_{3}+C_{4}\right) I^{2}-113 I+C_{1} C_{2} W+300=0
$$

\section{References}

[1] Pahl, G., and Beitz, W., 1996, Engineering Design: A Systematic Approach, K. Wallace, ed., Springer, London.

[2] Asimow, M., 1962, Introduction to Design, Prentice-Hall, Englewood Cliffs, NJ.

[3] Ullman, D. G., 2003, The Mechanical Design Process, 3rd ed., McGraw-Hill, Boston.

[4] Ulrich, K. T., and Eppinger, S. D., 2004, Product Design and Development, 3rd ed., McGraw-Hill, Irwin, NY.

[5] Cooper, R. G., 1994, "Third-Generation New Product Processes," J. Prod. Innovation Manage., 11(1), pp. 3-14.

[6] Cooper, R. G., 2008, "The Stage-Gate Idea-to-Launch Process-Update, What's New, and NexGen Systems," J. Prod. Innovation Manage., 25(3), pp. $213-232$.

[7] Herrmann, J. W., and Schmidt, L. C., 2002, "Viewing Product Development as a Decision Production System," ASME Paper No. DETC2002/DTM-34030.

[8] Herrmann, J. W., and Schmidt, L. C., 2006, "Product Development and Decision Production Systems," Decision Making in Engineering Design, K. Lewis, W. Chen, and L. C. Schmidt, eds., ASME, New York.

[9] Lewis, K., and Mistree, F., 1998, "Collaborative, Sequential, and Isolated Decisions in Design," ASME J. Mech. Des., 120(4), pp. 643-652.

[10] Shiau, C.-S. N., and Michalek, J. J., 2009, "Should Designers Worry About Market Systems?," ASME J. Mech. Des., 131(1), p. 011011.

[11] Hazelrigg, G. A., 1998, "A Framework for Decision-Based Engineering Design,” ASME J. Mech. Des., 120, pp. 653-658.

[12] Renaud, J. E., and Gu, X., 2006, "Decision-Based Collaborative Optimization of Multidisciplinary Systems," Decision Making in Engineering Design, K. Lewis, W. Chen, and L. C. Schmidt, eds., ASME, New York, pp. 173-186.

[13] Michalek, J. J., Ceryan, O., Papalambros, P. Y., and Koren, Y., 2006, "Balancing Marketing and Manufacturing Objectives in Product Line Design," ASME J. Mech. Des., 128(6), pp. 1196-1204.

[14] Williams, N., Azarm, S., and Kannan, P. K., 2008, "Engineering Product Design Optimization for Retail Channel Acceptance," ASME J. Mech. Des., 130(6), p. 061402

[15] Smith, P. G., and Reinersten, D. G., 1991, Developing Products in Half the Time, Van Norstrand Reinhold, New York.

[16] O’Donovan, B., Eckert, C., Clarkson, J., and Browning, T. R., 2005, "Design Planning and Modelling," Design Process Improvement: A Review of Current Practice, J. Clarkson and C. Eckert, eds., Springer, London, pp. 60-87.

[17] Adler, P. S., Mandelbaum, A., Nguyen, V., and Schwerer, E., 1995, "From Project to Process Management: An Empirically-Based Framework for Analyzing Product Development Time," Manage. Sci., 41(3), pp. 458-484.

[18] Johnson, E. W., and Brockman, J. B., 1998, "Measurement and Analysis of Sequential Design Processes," ACM Trans. Des. Autom. Electron. Syst., 3(1), pp. 1-20.

[19] Browning, T. R., 2001, "Applying the Design Structure Matrix to System Decomposition and Integration Problems: a Review and New Directions," IEEE Trans. Eng. Manage., 48(3), pp. 292-306.

[20] O'Donovan, B., Eckert, C., and Clarkson, P. J., 2004, "Simulating Design Processes to Assist Design Process Planning," ASME Paper No. DETC200457612.

[21] Cho, S.-H., and Eppinger, S. D., 2005, "A Simulation-Based Process Model for Managing Complex Design Projects,” IEEE Trans. Eng. Manage., 52(3), pp. $316-328$.

[22] Wynn, D. C., and John Clarkson, P., 2009, "Design Project Planning, Monitoring and Re-Planning Through Process Simulation," International Conference on Engineering Design, ICED'09, Stanford University, Stanford, CA, Aug. 24-27.

[23] Eckert, C. M., and Clarkson, P. J., 2003, "The Reality of Design Process Planning," International Conference on Engineering Design, ICED'03, Stockholm, Sweden.

[24] Chang, T.-S., and Ward, A. C., 1995, "Conceptual Robustness in Simultaneous Engineering: A Formulation in Continuous Space,” Res. Eng. Des., 7(2), pp. $67-85$.

[25] Krishnan, V. D., and Eppinger, S. D., and Whitney, D. E., 1992, "Ordering Cross-Functional Decision Making in Product Development," Working Paper
No. 3299-91, https://dspace.mit.edu/bitstream/1721.1/2360/1/SWP-329924063233.pdf

[26] Krishnan, V., Eppinger, S. D., and Whitney, D. E., 1997, "Simplifying Iterations in Cross-Functional Design Decision Making," ASME J. Mech. Des., 119, pp. 485-493.

[27] Karimian, P., and Herrmann, J. W., 2009, "Separating Design Optimization Problems to Form Decision-Based Design Processes," ASME J. Mech. Des. 131(1), p. 011007.

[28] Gurnani, A., and Lewis, K., 2008, "Collaborative, Decentralized Engineering Design at the Edge of Rationality," ASME J. Mech. Des., 130(12), p. 121101.

[29] Schoeffler, J. D., 1971, "Static Multilevel Systems," Optimization Methods for Large-Scale Systems, D. A. Wismer, ed., McGraw-Hill, New York, pp. 1-47.

[30] Kirsch, U., 1981, Optimum Structural Design, McGraw-Hill, New York.

[31] Kim, H., Ragon, S., Soremekun, G., and Malone, B., 2004, "Flexible Approximation Model for Bi-Level Integrated System Synthesis," Tenth AIAA/ISSMO Multidisciplinary Analysis and Optimization Conference, Albany, NY, Aug 30-Sep. 1, Paper No. AIAA 2004-4545.

[32] Kim, H. M., Michelena, N. F., Papalambros, P. T., and Jiang, T., 2000, "Target Cascading in Optimal System Design," Proceedings of DETC 2000, 26th Design Automation Conference, Baltimore, MD, Sep. 10-13, Paper No. DETC2000/DAC-14265.

[33] Kim, H. M., Rideout, D. G., Papalambros, P. Y., and Stein, J. L., 2003, “Analytical Target Cascading in Automotive Vehicle Design,” ASME J. Mech. Des., 125(3), pp. 481-489.

[34] Tosserams, S., Kokkolaras, M., Etman, L. F. P., and Rooda, J. E., 2008, "Extension of Analytical Target Cascading Using Augmented Lagrangian Coordination for Multidisciplinary Design Optimization," Twelfth AIAA/ISSMO Multidisciplinary Analysis and Optimization Conference, Victoria, BC, Canada, Sep. 10-12.

[35] Bloebaum, C. L., Hajela, P., and Sobieszczanski-Sobieski, J., 1992, "NonHierarchic System Decomposition in Structural Optimization,” Eng. Optimiz., 19(3), pp. 171-186

[36] Wujek, B. A., Renaud, J. E., Batill, S. M., and Brockman, J. B., 1996, "Concurrent Subspace Optimization Using Design Variable Sharing in a Distributed Computing Environment," Concurrent Engineering," Research and Applications, 4(4), pp. 361-377.

[37] Chen, L., and Li, S., 2001, "Concurrent Parametric Design Using a Multifunctional Team Approach," ASME Paper No. DAC-21038.

[38] Simon, H. A., 1997, Models of Bounded Rationality, MIT Press, Cambridge, MA, Vol. 3 .

[39] Simon, H. A., 1997, Administrative Behavior, 4th ed., Free, New York.

[40] Gerd, G., and Todd, P. M., 1999, Simple Heuristics That Make Us Smart, Oxford University Press, New York.

[41] March, J., and Simon, H., 1993, Organizations, 2nd ed., Blackwell, Cambridge, MA.

[42] Dym, C. L., Wood, W. H., and Scott, M. J., 2002, "Rank Ordering Engineering Designs: Pairwise Comparison Charts and Borda Counts," Res. Eng. Des. 13(4), pp. 236-242.

[43] Mintzberg, H., Raisinghani, D., and Theoret, A., 1976, "The Structure of Unstructured Decision Processes," Adm. Sci. Q., 21(2), pp. 246-275.

[44] Nutt, P. C., 2005, "Search During Decision-Making," Eur. J. Oper. Res., 160, pp. 851-876.

[45] Wang, Y., and Chiew, V., 2008, "On the Cognitive Process of Human Problem Solving,” Cogn. Syst. Res., 11, pp. 81-92.

[46] Simpson, T. W., 1998, "A Concept Exploration Method for Product Family Design," Ph.D. thesis, Georgia Institute of Technology, Atlanta, GA.

[47] Simpson, T. W., 2006, "Methods for Optimizing Product Platforms and Product Families," Product Platform and Product Family Design: Methods and Applications, T. W. Simpson, Z. Siddique, and J. Jiao, eds., Springer, New York.

[48] Wassenaar, H. J., Kumar, D., and Chen, W., 2006, "Discrete Choice Demand Modeling for Decision-Based Design," Decision Making in Engineering Design, K. Lewis, W. Chen, and L. C. Schmidt, eds., ASME, New York.

[49] Wassenaar, H. J., and Chen, W., 2001, "An Approach to Decision-Based Design," ASME Paper No. DETC01/DTM-21683.

[50] Simon, H. A., 1981, The Sciences of the Artificial, 2nd ed., MIT Press, Cambridge, MA

[51] Sandberg, J., 2001, "Understanding Competence at Work," Harvard Bus. Rev., 73(3), pp. 24-28. 